\title{
Intrathecal Analgesia and Restrictive Perioperative Fluid Management within Enhanced Recovery Pathway: Hemodynamic Implications
}

\author{
Martin Hübner, MD, Jenna K Lovely, PharmD, BCPS, Marianne Huebner, PhD, Seth W Slettedahl, MS, \\ Adam K Jacob, MD, MS, David W Larson, MD, MBA, FACS, FASCRS
}

BACKGROUND: Intrathecal analgesia and avoidance of perioperative fluid overload are key items within enhanced recovery pathways. Potential side effects include hypotension and renal dysfunction.

STUDY DESIGN: From January 2010 until May 2010, all patients undergoing colorectal surgery within enhanced recovery pathways were included in this retrospective cohort study and were analyzed by intrathecal analgesia (IT) vs none (noIT). Primary outcomes measures were systolic and diastolic blood pressure, mean arterial pressure, and heart rate for 48 hours after surgery. Renal function was assessed by urine output and creatinine values.

RESULTS: One hundred and sixty-three consecutive colorectal patients (127 IT and 36 noIT) were included in the analysis. Both patient groups showed low blood pressure values within the first 4 to 12 hours and a steady increase thereafter before return to baseline values after about 24 hours. Systolic and diastolic blood pressure and mean arterial pressure were significantly lower until 16 hours after surgery in patients having IT compared with the noIT group. Low urine output $(<0.5 \mathrm{~mL} / \mathrm{kg} / \mathrm{h})$ was reported in $11 \%$ vs $29 \%$ (IT vs noIT; $\mathrm{p}=0.010$ ) intraoperatively, $20 \%$ vs $11 \%(\mathrm{p}=0.387), 33 \%$ vs $22 \%(\mathrm{p}=0.304)$, and $31 \%$ vs $21 \%$ $(\mathrm{p}=0.478)$ for postanesthesia care unit and postoperative days 1 and 2 , respectively. Only 3 of $127(2.4 \%)$ IT and 1 of $36(2.8 \%)$ noIT patients had a transitory creatinine increase $>50 \%$; no patients required dialysis.

CONCLUSIONS: Postoperative hypotension affects approximately $10 \%$ of patients within an enhanced recovery pathway and is slightly more pronounced in patients with IT. Hemodynamic depression persists for $<20$ hours after surgery; it has no measurable negative impact and therefore cannot justify detrimental postoperative fluid overload. (J Am Coll Surg 2013; 216:1124-1134. (C) 2013 by the American College of Surgeons)

Enhanced recovery pathways (ERPs) successfully reduce complications, hospital stay, and costs in colorectal surgery and are considered standards of care in many centers. ${ }^{1-5}$ Two of the key principles of ERPs are the

Disclosure Information: Nothing to disclose.

Presented to the European Society of Coloproctology, Vienna, Austria, September 2012.

Received December 16, 2012; Revised February 10, 2013; Accepted February 15, 2013.

From the Department of Surgery, Division of Colon and Rectal Surgery (Hübner, Larson), Hospital Pharmacy Services (Lovely), Division of Biostatistics (Huebner, Slettedahl), and Department of Anesthesiology (Jacob), Mayo Clinic, Rochester, MN, and Department of Visceral Surgery (Hübner), University Hospital CHUV, Lausanne, Switzerland.

Correspondence address: David W Larson, MD, MBA, FACS, FASCRS, Department of Surgery, Division of Colon and Rectal Surgery, Mayo Clinic, 200 First St SW, Rochester, MN 55905. email: larson.david2@mayo.edu avoidance of perioperative fluid overload and minimization of systemic opioid consumption. ${ }^{4,6-9}$

Optimal perioperative fluid management within an ERP aims to maintain homeostasis and has become more restrictive than traditional fluid management. ${ }^{4,6,9-13}$ Modern protocols propagate a zero fluid balance. ${ }^{14}$ Traditional fluid management by contrast aims to maintain blood pressure (BP) and heart rate (HR) by liberal administration of IV fluids to prevent the potential for hypovolemia-induced disturbance of microcirculation and organ dysfunction. ${ }^{12,15}$ Evidence for a more stringent fluid administration is compelling, as the results of this strategy lead to reduced cardiopulmonary complications and faster intestinal recovery without compromising wound healing. ${ }^{4,7,9,10,12,16-20}$

Current European recommendations propagate midthoracic epidural analgesia (EDA) as the perioperative analgesic mainstay in ERP to limit use of systemic opioids 


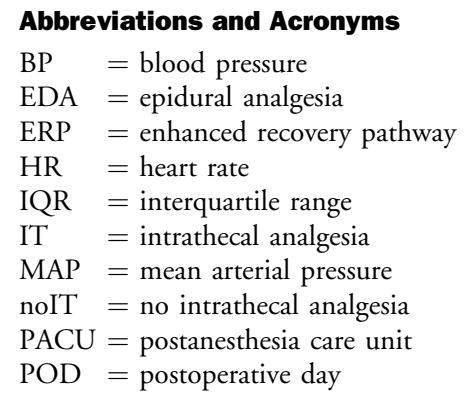

and, specifically, their related side effects. ${ }^{9}$ The beneficial effects of EDA on pain reduction, intestinal recovery, and pulmonary morbidity have been widely acknowledged. ${ }^{21-29}$ Potential side effects of EDA include transient arterial hypotension in about $10 \%$ of patients, pruritus, and urinary complications. ${ }^{24,28-31}$ In addition, EDA requires postoperative follow-up and specialized nursing care, making it work- and cost-intensive. In addition, the patient is bound to an additional device, which can impede early ambulation. Recent studies have suggested that, in the setting of ERP, single-injection intrathecal analgesia (IT) can provide equivalent analgesia compared with EDA, but with earlier return of bowel function, earlier mobility, and shorter hospital length of stay after elective laparoscopic colorectal surgery. ${ }^{32-35}$

Balanced fluid management, with the goal of euvolemic state, and neuraxial blockade both have the potential to induce arterial hypotension and hypoperfusion-induced organ dysfunction, such as renal insufficiency, myocardial ischemia, and impaired wound healing. ${ }^{12,15}$ Episodic hypotension, whether the result of IT or fluid restriction, can result in excess fluid administration with the intention to "correct" a perceived "hypovolemic state."

The objective of this retrospective cohort study is to test the hypothesis that the combination of balanced IV fluid management in conjunction with IT adversely affects perioperative hemodynamics and renal function.

\section{METHODS}

After obtaining Mayo Clinic Institutional Review Board approval, all consecutive patients undergoing elective open and minimally invasive colorectal procedures within a standardized ERP at Mayo Clinic, Rochester between January 2010 and May 2010 were identified. Patients that failed to provide research authorization were excluded. Patients with IT were compared with patients without (noIT). All data were collected from electronic medical records and the Colorectal Surgery Division Database with prospectively defined variables and were continuously maintained by research staff unaware of the study hypothesis.

\section{Enhanced recovery pathway}

This institution's ERP was implemented in November 2009 and initial results have been described in detail previously. ${ }^{5}$ Briefly, pain management includes preoperative treatment with celecoxib and gabapentin, intraoperative IT, and scheduled acetaminophen and nonsteroidal antiinflammatory medications postoperatively. In addition, oral oxycodone was available postoperatively as needed for reported numeric pain intensity score rating $>4$. Intraoperative fluid guidelines were set, with laparoscopic operative patients to receive $5 \mathrm{~mL} / \mathrm{kg} / \mathrm{h}$ or up to $500 \mathrm{~mL} / \mathrm{h}$ and open procedural patients to receive $8 \mathrm{~mL} / \mathrm{kg} / \mathrm{h}$ (up to $800 \mathrm{~mL} / \mathrm{h}$ ). Intraoperative fluid administration consisted of lactated Ringer's solution and an additional $500 \mathrm{~mL}$ of $5 \%$ albumin if the anesthetist believed it to be necessary on evaluation of intravascular status. Postoperative IV fluids were, by default, dextrose $5 \%$ and $0.45 \%$ sodium chloride with $20 \mathrm{mEq} / \mathrm{L}$ potassium chloride. Postoperative maintenance rate of $40 \mathrm{~mL} / \mathrm{h}$ was to be continued until the morning after surgery (POD1) at $8 \mathrm{AM}$. Intraoperatively, all patients underwent general endotracheal anesthesia with an inhaled anesthetic in air/oxygen. All patients received postoperative nausea and vomiting prophylaxis consisting of dexamethasone and a 5HT-3 antagonist plus droperidol for patients at highest risk based on institutional postoperative nausea and vomiting consensus guidelines. The nasogastric tube was removed at the end of the procedure and oral intake (eg, fluids, oral nutritional supplements, regular diet) was started 4 hours after surgery. Patients were assisted by dedicated nursing staff to be mobilized 2 hours on the day of surgery and 8 hours per day on the following days. The urinary catheter was removed the day after surgery at 8 AM. Discharge criteria include adequate pain control, oral intake, and return to baseline mobility. Resumption of bowel movement was not a compulsory requirement for discharge.

\section{Intrathecal analgesia}

As part of the ERP, patients were consented for singleinjection IT placed under sterile conditions immediately before induction of general anesthesia. Patients in whom neuraxial regional anesthesia was contraindicated (eg, patients with coagulopathy or pre-existing neurologic dysfunction) or who refused regional anesthesia were given IV systemic opiates intraoperatively. The IT injection consisted of preservative-free hydromorphone ( 0.05 to $0.08 \mathrm{mg}$ ) with intrathecal bupivacaine or clonidine adjuncts given at the discretion of the attending anesthesiologist. Administration of additional systemic opiates intraoperatively and in the postanesthesia care unit (PACU) was at the discretion of the attending anesthesiologist. 


\section{Outcomes measures}

Demographics, diagnosis, and surgical details were collected as potential confounders. Similarly, intra- and postoperative IV and oral fluids were collected along with daily weight measurements.

Outcomes of primary interest were postoperative values for systolic and diastolic BP, mean arterial pressure (MAP), and $\mathrm{HR}$ in patients that received IT compared with patients that did not receive IT. For the purpose of this study, measurements starting from the time in the recovery unit (PACU) at 4-hour intervals until 48 hours after return to room were considered. Hemodynamic instability was defined as a patient experiencing one or more of the following: systolic BP $<90 \mathrm{mmHg}$, MAP <60 mmHg, or HR >100 bpm.

Secondarily, kidney function was assessed by urine output per day until 48 hours after return to room and reported as absolute values and adjusted per body weight and time. Special attention was given to patients presenting postoperative oliguria, defined as $<0.5 \mathrm{~mL} / \mathrm{kg} / \mathrm{h}$. In addition, perioperative serum creatinine values were used to assess for risk of renal dysfunction according to the Acute Kidney Injury Network classification system. ${ }^{36}$

All postoperative complications (ie, ileus, bowel obstruction, anastomotic leak, wound infection/abscess, venous thromboembolism, atelectasis/respiratory dysfunction, acute renal failure, urinary retention, and urinary tract infection) were collected until 30 days after surgery. Length of hospital stay was counted from the date of surgery to the date of discharge. Postoperative bowel recovery was defined as time to first stool/flatus with resumption of a regular diet. Postoperative ileus was counted from POD2 on and was defined as intolerance to oral intake and nausea/vomiting requiring reinsertion of a nasogastric tube.

\section{Statistical analysis}

Descriptive statistics for categorical variables were reported as frequency and percentage, and continuous variables were reported as mean (SD) or median (interquartile range), as appropriate. Continuous variables were compared between patients with or without intrathecal use with the Wilcoxon rank sum test or $t$-test as appropriate. Chi-square or Fisher's exact test were used for comparison of categorical variables.

All statistical tests were 2-sided and a level of 0.05 was considered statistically significant. Data analyses were performed using SAS software version 9.2 (SAS Institute).

\section{RESULTS}

Overall, 163 consecutive patients were included in this retrospective cohort study. Demographic information is presented in Table 1. Within the standardized clinical pathway, ERP, 127 of 163 (78\%) patients received IT, and IT was contraindicated or refused in $36(22 \%)$ patients. In most patients, intrathecal injections contained hydromorphone, bupivacaine, and clonidine (84\%), and the remainder contained hydromorphone alone (3.2\%), hydromorphone plus bupivacaine $(3.2 \%)$, or hydromorphone plus clonidine (9.6\%). Overall, IT and noIT groups were well matched. A greater proportion of noIT patients were American Society of Anesthesiologists class 3 or 4 compared with IT patients (52.8\% vs $23.6 \%$; $\mathrm{p}=0.0008)$.

\section{Perioperative fluid-related parameters}

There was no significant difference in IV or PO fluid intake between IT and noIT groups for any of the given time points (Table 2). Median (interquartile range [IQR], ie, quartile 1 to 3 ) intraoperative fluid administration was higher than the a priori defined fluid goal, but not significantly different between IT (median $10.1 \mathrm{~mL} / \mathrm{kg} / \mathrm{h} ; \mathrm{IQR}$ 7.2 to $14.4 \mathrm{~mL} / \mathrm{kg} / \mathrm{h}$ ) and noIT (median $8.8 \mathrm{~mL} / \mathrm{kg} / \mathrm{h}$; IQR 6.5 to $14.1 \mathrm{~mL} / \mathrm{kg} / \mathrm{h})$ groups $(\mathrm{p}=0.341)$. Postoperative fluid administration was in agreement with the ERP protocol, with no significant differences between IT and noIT groups (Fig. 1A). Of note, only 29 of 163 patients $(17.8 \%)$ had not discontinued all IV fluids on POD2.

A moderate postoperative weight gain was documented for both groups. Compared with their preoperative weight, IT and noIT groups increased their weight by $2.0 \pm 2.4 \mathrm{~kg}$ vs $1.7 \pm 1.8 \mathrm{~kg}(\mathrm{p}=0.396)$ on POD1. The change from baseline weight had decreased by POD2, $1.3 \pm 5.2 \mathrm{~kg}$ vs $1.5 \pm 3.3 \mathrm{~kg}$ for IT and noIT groups, respectively ( $\mathrm{p}=0.654)$.

The total urine output for the intra- and postoperative time were similar between IT and noIT patients (Table 2). There was no significant difference for weightand time-adjusted urine output between IT and noIT patients as displayed in Figure 1B. However, a significant proportion had low urine output $(<0.5 \mathrm{~mL} / \mathrm{kg} / \mathrm{h}): 11 \%$ vs $29 \%$ for IT vs noIT intraoperatively ( $p=0.010), 20 \%$ vs $11 \%(\mathrm{p}=0.252)$ PACU, 33\% vs $22 \%(\mathrm{p}=0.304)$ POD 1 , and $31 \%$ vs $21 \%(\mathrm{p}=0.478)$ POD2. Acute renal failure developed in 3 of 127 (2.4\%) IT patients and 1 of 36 $(2.8 \%)$ noIT patients, according to the applied definition $(\mathrm{p}=1.000)$; all 4 patients were asymptomatic and recovered rapidly and without any sequelae or dialysis.

\section{Postoperative hemodynamics}

After surgery, patients were brought to the recovery room (PACU) until stable for return to their room. Mean time spent in the PACU was not significantly different for IT and noIT groups (Table 2). Figure 2 illustrates systolic and diastolic BP, MAP, and HR for the postoperative period starting in the PACU until 48 hours after return to room. In the PACU and at return to room, systolic and diastolic 
Table 1. Demographics and Operation Characteristics

\begin{tabular}{|c|c|c|c|c|}
\hline Variable & $\begin{array}{l}\text { Intrathecal anesthesia } \\
\text { used }(n=127)\end{array}$ & $\begin{array}{c}\text { No intrathecal anesthesia } \\
\text { used }(n=36)\end{array}$ & $\begin{array}{c}\text { Total } \\
(\mathrm{n}=163)\end{array}$ & p Value \\
\hline Sex, n (\%) & & & & 0.661 \\
\hline Female & $51(40.2)$ & $13(36.1)$ & $64(39.3)$ & \\
\hline Male & $76(59.8)$ & $23(63.9)$ & $99(60.7)$ & \\
\hline Age, y, mean (SD) & $52.5(17.7)$ & $54.1(18.7)$ & $52.8(17.9)$ & \\
\hline ASA, n (\%) & & & & 0.003 \\
\hline 1 & $9(7.1)$ & $2(5.6)$ & $11(6.7)$ & \\
\hline 2 & $88(69.3)$ & $15(41.7)$ & $103(63.2)$ & \\
\hline 3 & $30(23.6)$ & $18(50.0)$ & $48(29.4)$ & \\
\hline 4 & $0(0.0)$ & $1(2.8)$ & $1(0.6)$ & \\
\hline BMI, mean (SD) & $27.1(6.1)$ & $28.1(5.2)$ & $27.3(5.9)$ & \\
\hline Diagnosis, n (\%) & & & & 0.969 \\
\hline Neoplasia & $45(35.4)$ & $13(37.1)$ & $58(35.8)$ & \\
\hline Diverticulitis & $12(9.4)$ & $4(11.4)$ & $16(9.9)$ & \\
\hline IBD & $41(32.3)$ & $10(28.6)$ & $51(31.5)$ & \\
\hline Misc & $29(22.8)$ & $8(22.9)$ & $37(22.8)$ & \\
\hline Procedure, n (\%) & & & & 0.068 \\
\hline Segmental resection* & $32(25.2)$ & $12(33.3)$ & $44(27.0)$ & \\
\hline Rectum/(sub)total & $36(28.3)$ & $7(19.4)$ & $43(26.4)$ & \\
\hline Ostomy creation/closure & $33(26.0)$ & $4(11.1)$ & $37(22.7)$ & \\
\hline Miscellaneous & $26(20.5)$ & $13(36.1)$ & $39(23.9)$ & \\
\hline Surgical approach, n (\%) & & & & 0.183 \\
\hline Open & $78(61.4)$ & $17(47.2)$ & $95(58.3)$ & \\
\hline Laparoscopic & $49(38.6)$ & $19(52.8)$ & $68(41.7)$ & \\
\hline EBL, mL, median (IQR) & $100(25-200)$ & $50(27.5-225)$ & $75(25-200)$ & \\
\hline Surgery duration, min, mean (SD) & $179.9(87.6)$ & $158.1(100.8)$ & $175.1(90.8)$ & \\
\hline PACU duration, min, mean (SD) & $150.5(65.9)$ & $142.5(65.1)$ & $148.7(65.6)$ & \\
\hline
\end{tabular}

*Segmental resections include ileocecal resections, (extended) right and left colectomies as well as transverse and sigmoid resections.

ASA, American Society of Anesthesiologists; BMI, body mass index (calculated as kg/m²); IBD, inflammatory bowel disease; EBL, estimated blood loss; IQR, interquartile range (quartile 1 to 3 ); PACU, postanesthesia care unit (recovery room).

$\mathrm{BP}$ and MAP were significantly lower in the IT group compared with noIT; and compared with a PACU baseline value, both patient groups showed a marked drop in BP within the first 4 to 12 hours and a steady increase thereafter before return to normal after about 24 hours. Heart rate remained largely unaffected during this period.

Figure 3 illustrates the proportion of IT and noIT patients fulfilling at least one of the institutional criteria for hemodynamic instability. Overall, up to $10 \%$ of the patients had low systolic BP $(<90 \mathrm{mmHg})$ until 12 hours, but $\leq 2 \%$ after 24 hours. Systolic hypotension was more prevalent in the IT group ( $\mathrm{p}=0.045$ at 4 hours). Mean arterial pressure was $<60 \mathrm{mmHg}$ in 7 patients $(4.3 \%)$ at 12 hours; all patients had received IT. Transient tachycardia was more prevalent in patients without IT (25\%) in the first 24 hours after surgery, and no more than $10 \%$ of IT patients were affected at any given time point. Statistically significant differences with regard to tachycardia were found between IT and noIT patients in the PACU
( $\mathrm{p}=0.014)$ and 8 hours after return to room $(\mathrm{p}=0.015)$ in favor of lower heart rate in IT patients.

\section{Functional recovery, complications, and length of stay}

There were no significant differences between the comparative groups for any of the documented surgical and medical complications (Table 3). A regular diet was offered to all patients on POD0 according to the protocol. Oral intake was actually tolerated by $81 \%$ of IT patients and $85 \%$ of noIT patients on POD2 ( $\mathrm{p}=0.606)$. Return of bowel function occurred at a median of 2 days after surgery and, again, there was no difference between patients with or without IT. Urinary retention requiring reinsertion of the urinary catheter occurred in 3 patients of each group (2 vs $9 \% ; \mathrm{p}=0.121)$.

Length of stay was a median of 2 days (IQR 2 to 4 days) for IT patients and 3 days (IQR 2 to 4 days) for noIT patients $(\mathrm{p}=0.243)$. 
Table 2. Perioperative Fluid Administration and Urine Output

\begin{tabular}{|c|c|c|c|c|}
\hline Variable & $\begin{array}{l}\text { Intrathecal anesthesia } \\
\text { used }(n=127)\end{array}$ & $\begin{array}{c}\text { No intrathecal anesthesia } \\
\text { used }(n=36)\end{array}$ & Total $(n=163)$ & p Value \\
\hline IV fluids, mL, OR & & & & 0.058 \\
\hline Median & 2,000 & 1,925 & 2,000 & \\
\hline IQR & $1,600-2,750$ & $1,075-2,350$ & $1,500-2,650$ & \\
\hline IV fluids, mL, PACU & & & & 0.952 \\
\hline Median & 350 & 325 & 350 & \\
\hline IQR & $200-775$ & $200-825$ & $200-800$ & \\
\hline IV fluids, mL, POD0 & & & & 0.915 \\
\hline Median & 300 & 300 & 300 & \\
\hline IQR & $150-450$ & $150-600$ & $150-460$ & \\
\hline IV fluids, mL, POD1 & & & & 0.368 \\
\hline Median & 530 & 388 & 500 & \\
\hline IQR & $190-900$ & $150-963$ & $150-900$ & \\
\hline IV fluids, mL, POD2* & & & & 0.537 \\
\hline Median & 0 & 0 & 0 & \\
\hline IQR & $0-300$ & $0-400$ & $0-300$ & \\
\hline PO fluids, mL, POD0 & & & & 0.316 \\
\hline Median & 360 & 300 & 360 & \\
\hline IQR & $195-640$ & $180-500$ & $180-635$ & \\
\hline PO fluids, mL, POD1 & & & & 0.225 \\
\hline Median & 1,257 & 1,090 & 1,190 & \\
\hline IQR & $680-1,660$ & $478-1,520$ & $630-1,620$ & \\
\hline PO fluids, mL, POD2* & & & & 0.617 \\
\hline Median & 610 & 500 & 600 & \\
\hline IQR & $310-1,150$ & $420-960$ & $360-1,120$ & \\
\hline Urine output, mL, OR & & & & 0.109 \\
\hline Median & 225 & 160 & 210 & \\
\hline IQR & $100-400$ & $50-380$ & $100-400$ & \\
\hline Urine output, mL, PACU & & & & 0.271 \\
\hline Median & 110 & 125 & 115 & \\
\hline IQR & $65-190$ & $68-300$ & $65-210$ & \\
\hline 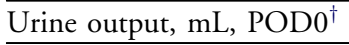 & & & & 0.019 \\
\hline Median & 300 & 450 & 325 & \\
\hline IQR & $200-469$ & $270-640$ & $210-500$ & \\
\hline Urine output, mL, POD1 & & & & 0.623 \\
\hline Median & 1,510 & 1,310 & 1,425 & \\
\hline IQR & $750-2,185$ & $800-2,445$ & $755-2,185$ & \\
\hline Urine output, mL, POD2* & & & & 0.103 \\
\hline Median & 1,155 & 1,576 & 1,250 & \\
\hline IQR & $675-1,673$ & $750-2,475$ & $700-1,715$ & \\
\hline
\end{tabular}

${ }^{*}$ Assessed until discharge.

${ }^{\dagger}$ Statistically significant $(\mathrm{p}<0.05)$.

IQR, interquartile range (quartile 1 to 3); NA, not available; OR, operation room; PACU, postanesthesia care unit (recovery room); PO, per oral; POD, postoperative day; RR, return to room.

\section{DISCUSSION}

Transient postoperative hemodynamic effects occur in patients within this ERP. These findings might be explained by our attempt at balanced fluid regimens or the use of IT (eg, bupivacaine and clonidine). However, the results of this study support the idea that there is no increased risk for hypovolemia-related complications. In addition, care providers should be permissive of 

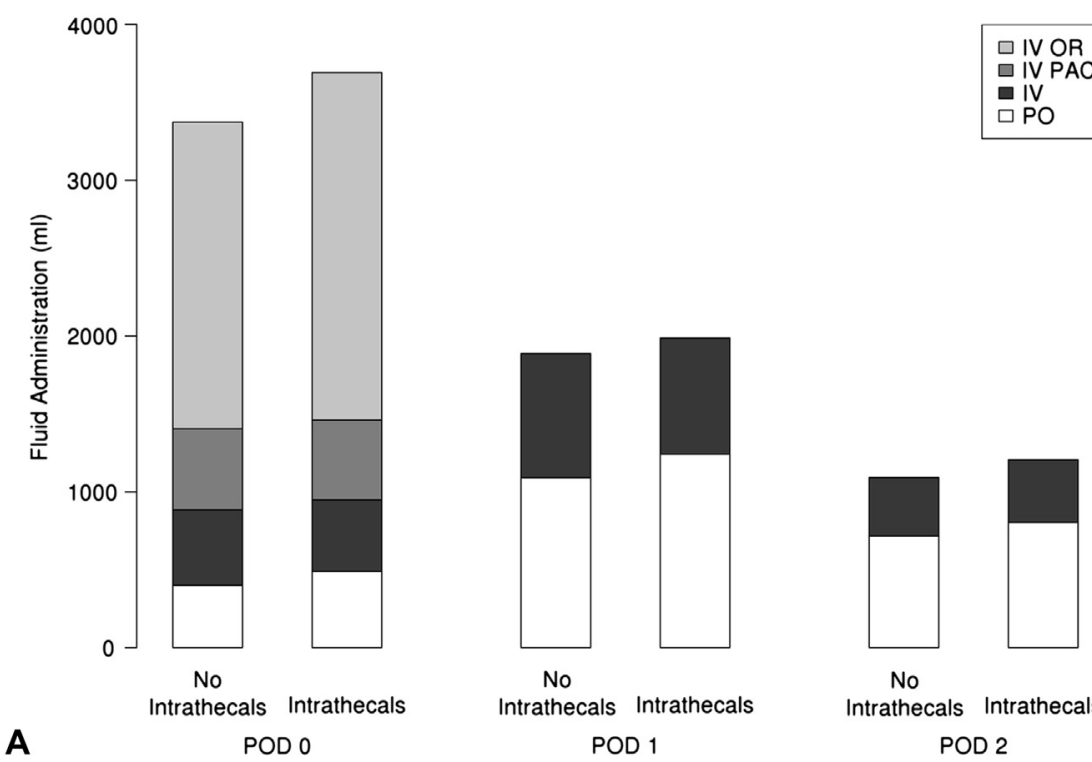

No Intrathecals Intrathecals POD 2

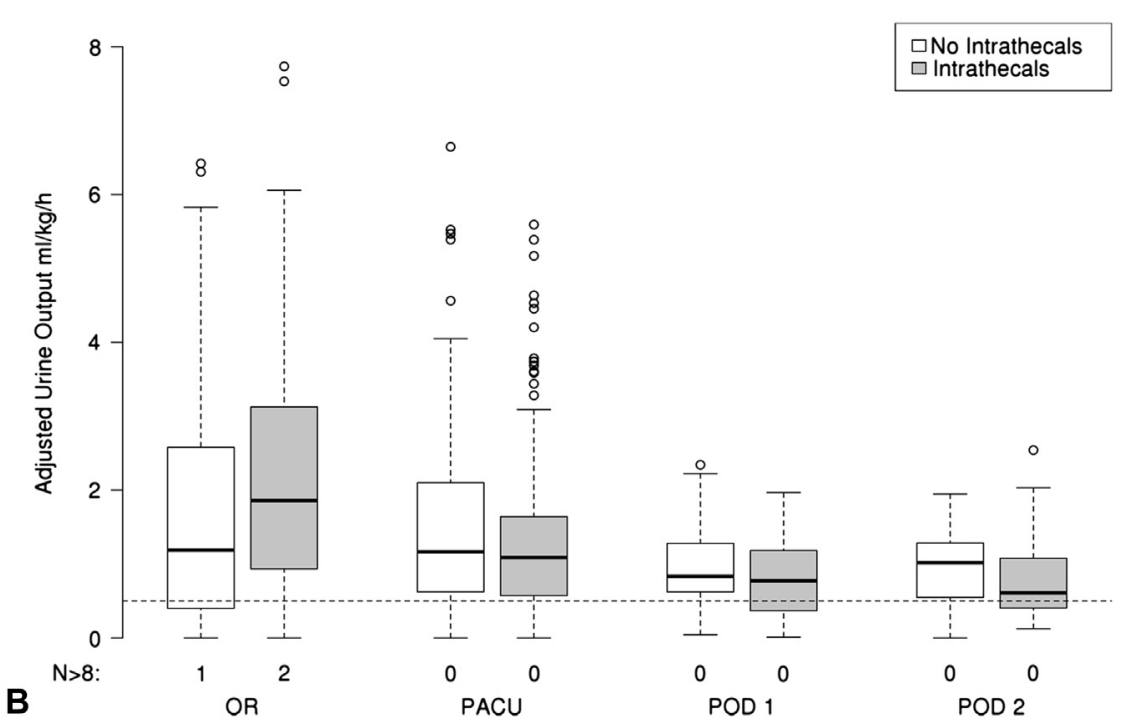

Figure 1. Perioperative fluid administration and urine output course. (A) Perioperative IV and per oral (PO) fluids. Perioperative fluid administration $(\mathrm{mL})$ on the day of surgery (POD0), POD1, and POD2 for patients with and without intrathecal analgesia. Oral intake was assessed until discharge. Postdischarge PO fluids for patients leaving on POD2 were not available. (B) Perioperative urine output. Weight- and time-adjusted urine output $(\mathrm{mL} / \mathrm{kg} / \mathrm{h})$ for patients with intrathecal (white bar) and those without (gray bar). The Box-and-Whisker plots display the median, interquartile range (Q1 to Q3), the range (3/2 times of interquartile range) and outliers. The dotted line indicates the threshold for low urine output $(<0.5 \mathrm{~mL} / \mathrm{kg} / \mathrm{h})$. OR, operating room; PACU, postanesthesia care unit.

transient hypotension because of the proven deleterious effects of postoperative fluid overload.

Perioperative fluid management is arguably one of the most important and controversial components of ERPs. There are abundant data suggesting that balanced fluid management to meet euvolemia is beneficial in terms of enhanced bowel recovery and reduced cardiopulmonary complications, overall complications, and hospital stay. ${ }^{9}, 10,12,16,17,37,38$ This also holds true for high-risk patients. $^{39}$ Transient hypotension is an acknowledged side effect of neuraxial blockade; postoperatively, about $10 \%-20 \%$ of patients are affected. ${ }^{27,30,32,33,40}$ In the 

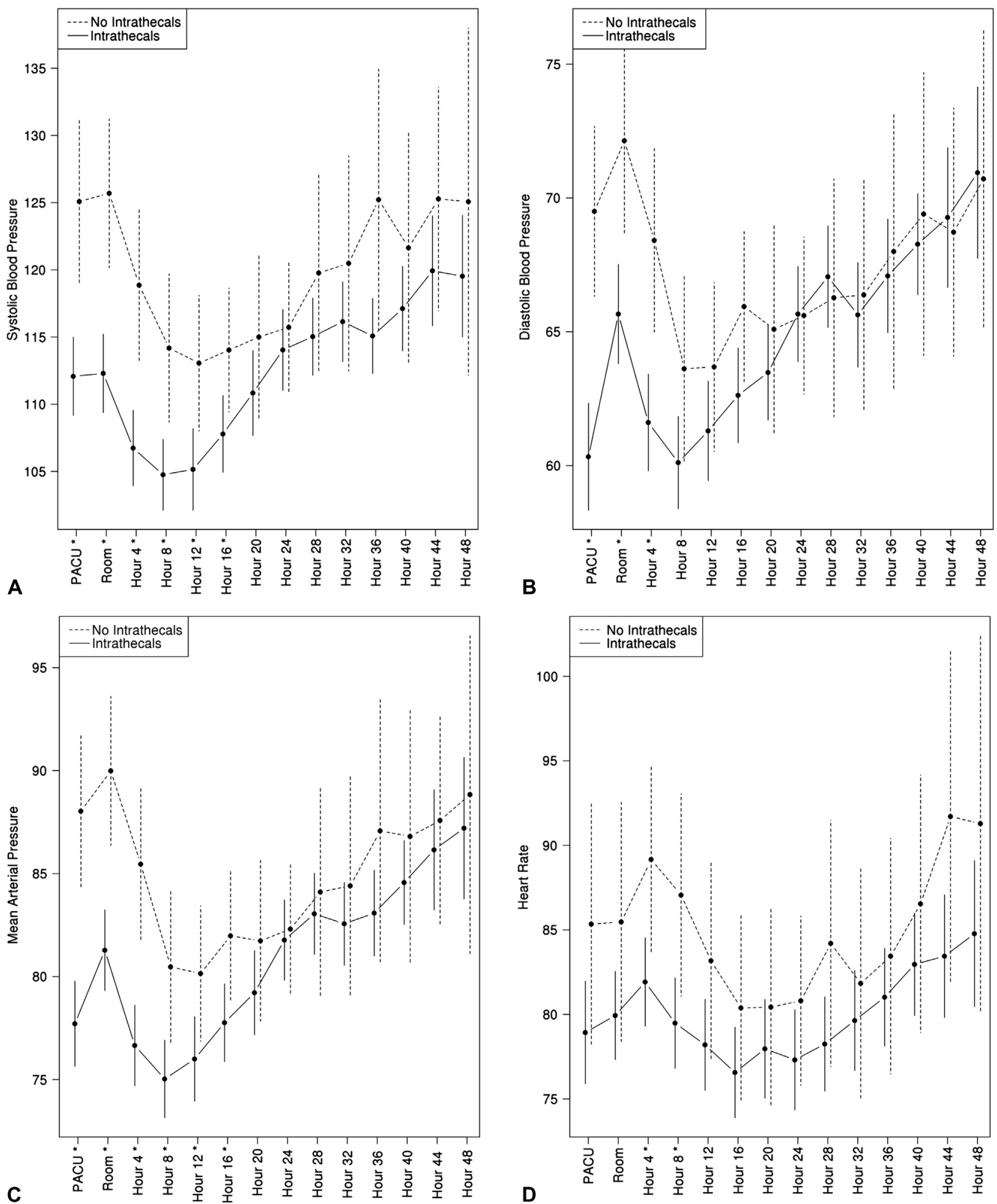

Figure 2. Postoperative hemodynamics. (A) Systolic blood pressure; (B) diastolic blood pressure; (C) mean arterial pressure; and (D) heart rate. *Statistical significance $(\mathrm{p}<0.05)$. OR, operating room; PACU, postanesthesia care unit. 
current series, hypotension occurred in patients with or without intrathecal injections (Fig. 3); hemodynamic depression was more common in the IT group yet transient, rarely below thresholds most would consider clinically relevant and without clinical consequences. Tachycardia occurred more frequently in the noIT group, which might be explained by stress due to a difference in pain-management technique.

Intra- and postoperative hypotension is a logical consequence that needs to be known and proactively planned for. Intraoperative hypotension is commonly treated with vasopressors (eg, phenylephrine) or small boluses of volume expanders. Esophageal Doppler-guided fluid administration has so far failed to translate into clinical benefits for the patient. ${ }^{14,41}$ Postoperative management is more challenging than intraoperative management; fluids are often given as a reactionary response to hypotension. ${ }^{40,42,43}$ Indeed, balanced fluid regimens seem to be a major hurdle in successful ERP implementation. ${ }^{43}$ This is of concern, as noncompliance with the ERP protocol, especially in the postoperative period, has clearly been related to worse outcomes, regardless of patient populations. ${ }^{2,39,42,43}$

Interestingly, the opposite held true in our series: postoperative IV fluid administration was very stringent and favored by excellent patient compliance with regard to oral intake starting the day of surgery (Fig. 1A). On the other hand, intraoperative fluid amounts were still rather traditional, which might be explained by the learning curve with the pathway itself. However, overall weight gain was minimal, with only about $1.5 \mathrm{~kg}$ on POD2, which demonstrates a nearly balanced fluid management that is perfectly in line with detailed reports on fluids from other centers. ${ }^{14}$

Low-dose vasopressor treatment has been advocated as an alternative treatment for hypotension in lieu of fluid administration, but concerns remain about end organ dysfunction and the cost associated with escalation of care needed for patients on these medications. These concerns have been quieted by our series and those of others. ${ }^{12,16,40} \mathrm{~A}$ recent meta-analysis of the available randomized trials on fluid management in colorectal surgery focused on the concept of the proper fluid balance between a real restriction on one side and fluid overload on the other side. ${ }^{13}$ Given our findings and those of others, transient hypotension and low urine output can be tolerated - when well understood and planned for-in the early postoperative period without any harm to the patient. In our experience, patients can be surveyed safely on the floor instead of with an overnight stay in a high-dependency unit, which has been advocated by others. ${ }^{30,40}$ However, indispensable requirements are a known perioperative clinical pathway, dedicated nursing teams, and well-trained residents and allied health staff who are able to assess volume status and who understand
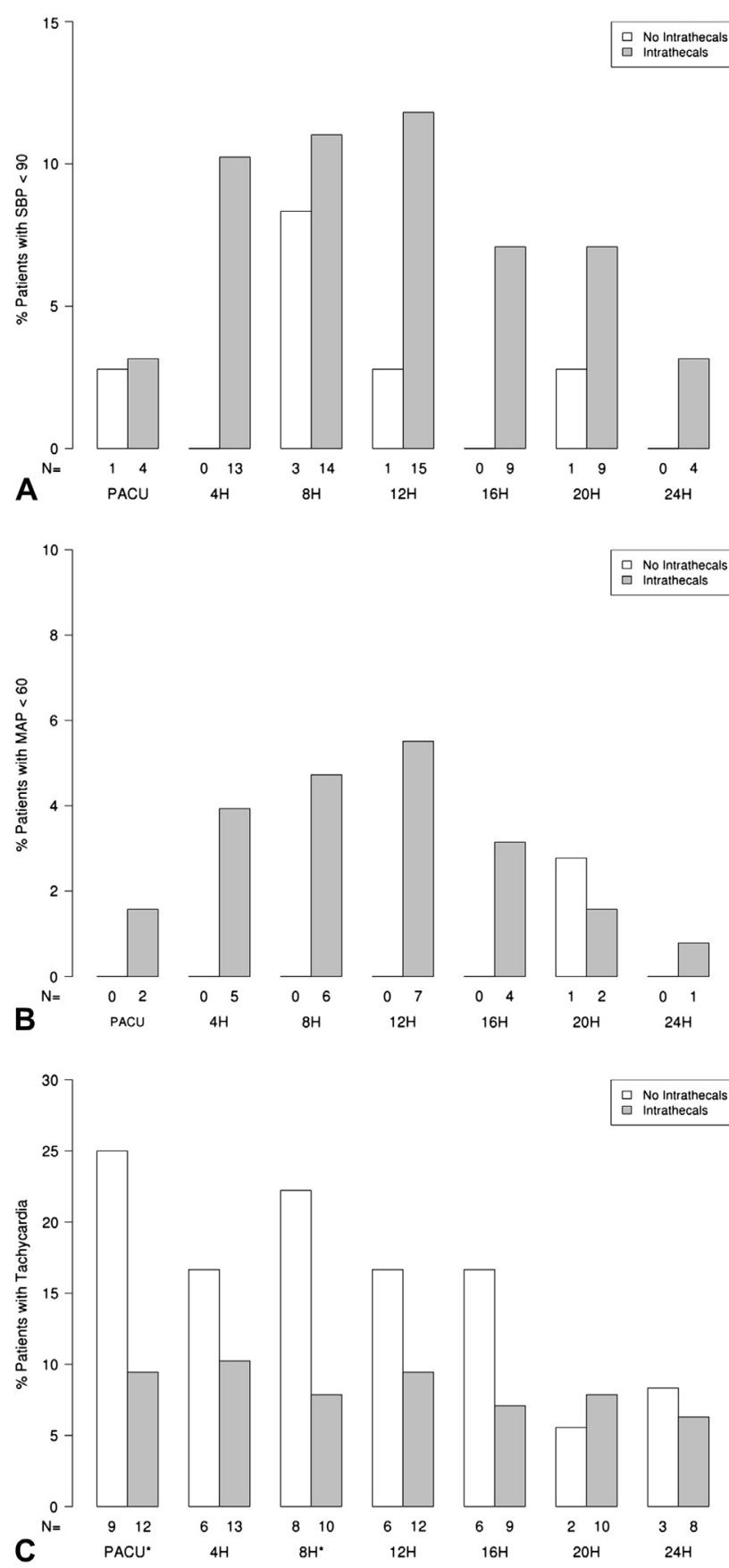

Figure 3. Hemodynamic instability in the postoperative period by intrathecals. (A) Patients with low systolic blood pressure (SBP) $<90 \mathrm{mmHg}$; (B) patients with low mean arterial pressure (MAP) <60 mmHg; (C) patients with tachycardia $>100 \mathrm{bpm}$, until 24 hours after return to room. *Statistical significance $(p<0.05)$. $\mathrm{PACU}$, postanesthesia care unit.

the goals for the patient within the pathway and the importance of avoiding fluid overload. When these requirements are fulfilled, excellent outcomes are possible. Tolerance of oral food, functional recovery, and discharge were achieved 
Table 3. Postoperative Complications

\begin{tabular}{|c|c|c|c|c|c|c|c|}
\hline \multirow[b]{2}{*}{ Complications } & \multicolumn{2}{|c|}{$\begin{array}{l}\text { Intrathecal anesthesia } \\
\text { used }(n=127)\end{array}$} & \multicolumn{2}{|c|}{$\begin{array}{c}\text { No intrathecal anesthesia } \\
\text { used }(n=36)\end{array}$} & \multicolumn{2}{|c|}{ Total $(n=163)$} & \multirow[b]{2}{*}{ p Value } \\
\hline & $\mathbf{n}$ & \%* & $\mathbf{n}$ & $\%$ * & $\mathbf{n}$ & $\%$ * & \\
\hline \multicolumn{8}{|l|}{$\underline{\text { Surgical complications }}$} \\
\hline Ileus & 26 & 20.8 & 4 & 12.1 & 30 & 19.0 & 0.325 \\
\hline Obstruction & 5 & 4.0 & 0 & & 5 & 3.1 & 0.585 \\
\hline Anastomotic leak/deep abscess & 5 & 3.9 & 1 & 2.8 & 6 & 3.7 & 0.744 \\
\hline Wound infection & 18 & 14.2 & 3 & 8.3 & 21 & 12.9 & 0.356 \\
\hline After leak/abscess & 5 & 3.9 & 1 & 2.8 & 6 & 3.7 & 0.744 \\
\hline After class II contamination & 12 & 9.4 & 2 & 5.6 & 14 & 8.6 & 0.462 \\
\hline After class III contamination & 1 & 0.8 & 0 & & 1 & 0.6 & 0.593 \\
\hline \multicolumn{8}{|l|}{ Medical complications } \\
\hline Venous thrombosis/embolism & 2 & 1.6 & 0 & & 2 & 1.3 & 1.000 \\
\hline Atelectasis/respiratory dysfunction & 4 & 3.2 & 1 & 2.9 & 5 & 3.1 & 1.000 \\
\hline Acute renal failure & 3 & 2.7 & 1 & 3.0 & 4 & 2.8 & 1.000 \\
\hline Urinary retention & 3 & 2.4 & 3 & 8.6 & 6 & 3.8 & 0.121 \\
\hline
\end{tabular}

*Percent of total $(\mathrm{n}=163)$.

Several complications are possible in the same patient. Clinical outcomes are assessed until 30 days after surgery.

in our series consistently after a median of 1,2 , and 3 days, respectively (current data and from Lovely ${ }^{5}$ ), and compare favorably with published data from other centers. ${ }^{3,44}$

Given the theoretical risk of urinary retention as a complication of neuraxial analgesia, clinicians have historically left urinary catheters in place longer than suggested by current recommendations. ${ }^{9}$ In our series, urinary retention was exceedingly rare in patients with and without IT. This is in accordance with other studies, ${ }^{40,45}$ and we suggest the removal of the urinary catheter on the morning of the day after surgery, as recommended in the guidelines mentioned. ${ }^{\text {? }}$

The current study is limited by its retrospective nature and nonmatched comparative groups. The IT patient group had a lower American Society of Anesthesiologists score, but underwent a higher percentage of major and open procedures; other potential confounders might be missing. In addition, definitive conclusions concerning all assessed outcomes measures are hampered by a relatively low study sample. Clearly, larger prospective datasets should be analyzed to reinforce our conclusions. On the other hand, we provide an extremely welldocumented consecutive series of ERP patients. To our knowledge, hemodynamic repercussions of IT and goaldirected fluid management have not been assessed in detail within ERPs. Our real-life experience adds to valid data to support the use of those combined measures.

\section{CONCLUSIONS}

Transient postoperative hypotension is prevalent in ERPs. Intraoperative fluid management and neuraxial analgesia can contribute to this condition. Along with these facts, low urine output can be anticipated in the first 24 hours and is therefore an unreliable indicator of fluid status. Therefore, a close clinical follow-up by a well-trained and proactive health care team is mandatory for the first day after surgery. Hemodynamic effects are transient and rarely reach a clinically relevant threshold beyond 20 hours after surgery. In addition, related complications such as renal dysfunction are exceedingly rare. Therefore, transient hypotension and lower but adequate urine output should be tolerated and liberal fluid administration in the postoperative period should be resisted so as not to jeopardize euvolemic state and its proven benefits for bowel recovery and overall clinical outcomes.

\section{Author Contributions}

Study conception and design: Hübner, Lovely, Jacob, Larson

Acquisition of data: Hübner, Lovely, Jacob

Analysis and interpretation of data: Hübner, Lovely, Huebner, Slettedahl, Jacob, Larson

Drafting of manuscript: Hübner, Lovely, Jacob, Larson Critical revision: Hübner, Lovely, Huebner, Slettedahl, Jacob, Larson

Acknowledgment: We extend our thanks to Deborah Attewell for assistance with data collection. We thank the nurses, pharmacists, anesthesiology teams, surgeons, and allied health staff who cared for the patients. We thank the Mayo Clinic Division of Colon and Rectal Surgery for funding the statistical analysis.

\section{REFERENCES}

1. Adamina M, Kehlet $H$, Tomlinson GA, et al. Enhanced recovery pathways optimize health outcomes and resource 
utilization: a meta-analysis of randomized controlled trials in colorectal surgery. Surgery 2011;149:830-840.

2. Muller S, Zalunardo MP, Hubner M, et al. A fast-track program reduces complications and length of hospital stay after open colonic surgery. Gastroenterology 2009;136: 842-847.

3. Varadhan KK, Neal KR, Dejong $\mathrm{CH}$, et al. The enhanced recovery after surgery (ERAS) pathway for patients undergoing major elective open colorectal surgery: a meta-analysis of randomized controlled trials. Clin Nutr 2010;29:434-440.

4. Wind J, Polle SW, Fung Kon Jin PH, et al. Systematic review of enhanced recovery programmes in colonic surgery. Br J Surg 2006;93:800-809.

5. Lovely JK, Maxson PM, Jacob AK, et al. Case-matched series of enhanced versus standard recovery pathway in minimally invasive colorectal surgery. Br J Surg 2012;99:120-126.

6. Fearon KC, Ljungqvist O, Von Meyenfeldt M, et al. Enhanced recovery after surgery: a consensus review of clinical care for patients undergoing colonic resection. Clin Nutr 2005;24: 466-477.

7. Kehlet H, Wilmore DW. Evidence-based surgical care and the evolution of fast-track surgery. Ann Surg 2008;248:189-198.

8. Weimann A, Braga M, Harsanyi L, et al. ESPEN guidelines on enteral nutrition: surgery including organ transplantation. Clin Nutr 2006;25:224-244.

9. Lassen K, Soop M, Nygren J, et al. Consensus review of optimal perioperative care in colorectal surgery: Enhanced Recovery After Surgery (ERAS) Group recommendations. Arch Surg 2009;144:961-969.

10. Brandstrup B, Tonnesen H, Beier-Holgersen R, et al. Effects of intravenous fluid restriction on postoperative complications: comparison of two perioperative fluid regimens: a randomized assessor-blinded multicenter trial. Ann Surg 2003;238:641-648.

11. Lobo DN. Fluid overload and surgical outcome: another piece in the jigsaw. Ann Surg 2009;249:186-188.

12. Rahbari NN, Zimmermann JB, Schmidt T, et al. Meta-analysis of standard, restrictive and supplemental fluid administration in colorectal surgery. Br J Surg 2009;96:331-341.

13. Varadhan KK, Lobo DN. A meta-analysis of randomised controlled trials of intravenous fluid therapy in major elective open abdominal surgery: getting the balance right. Proc Nutr Soc 2010;69:488-498.

14. Brandstrup B, Svendsen PE, Rasmussen M, et al. Which goal for fluid therapy during colorectal surgery is followed by the best outcome: near-maximal stroke volume or zero fluid balance? Br J Anaesth 2012;109:191-199.

15. Gan TJ, Soppitt A, Maroof M, et al. Goal-directed intraoperative fluid administration reduces length of hospital stay after major surgery. Anesthesiology 2002;97:820-826.

16. Holte K, Foss NB, Andersen J, et al. Liberal or restrictive fluid administration in fast-track colonic surgery: a randomized, double-blind study. Br J Anaesth 2007;99:500-508.

17. Lobo DN, Macafee DA, Allison SP. How perioperative fluid balance influences postoperative outcomes. Best Pract Res Clin Anaesthesiol 2006;20:439-455.

18. MacKay G, Fearon K, McConnachie A, et al. Randomized clinical trial of the effect of postoperative intravenous fluid restriction on recovery after elective colorectal surgery. Br J Surg 2006;93:1469-1474.

19. Marjanovic G, Villain C, Juettner E, et al. Impact of different crystalloid volume regimes on intestinal anastomotic stability. Ann Surg 2009;249:181-185.
20. Lobo DN, Bostock KA, Neal KR, et al. Effect of salt and water balance on recovery of gastrointestinal function after elective colonic resection: a randomised controlled trial. Lancet 2002;359:1812-1818.

21. Ahlers O, Nachtigall I, Lenze J, et al. Intraoperative thoracic epidural anaesthesia attenuates stress-induced immunosuppression in patients undergoing major abdominal surgery. $\mathrm{Br} \mathrm{J}$ Anaesth 2008;101:781-787.

22. Ali M, Winter DC, Hanly AM, et al. Prospective, randomized, controlled trial of thoracic epidural or patient-controlled opiate analgesia on perioperative quality of life. Br J Anaesth 2010;104:292-297.

23. Carli F, Mayo N, Klubien K, et al. Epidural analgesia enhances functional exercise capacity and health-related quality of life after colonic surgery: results of a randomized trial. Anesthesiology 2002;97:540-549.

24. Cashman JN, Dolin SJ. Respiratory and haemodynamic effects of acute postoperative pain management: evidence from published data. Br J Anaesth 2004;93:212-223.

25. Guay J. The benefits of adding epidural analgesia to general anesthesia: a metaanalysis. J Anesth 2006;20:335-340.

26. Hanna MN, Murphy JD, Kumar K, et al. Regional techniques and outcome: what is the evidence? Curr Opin Anaesthesiol 2009;22:672-677.

27. Kehlet H. Epidural analgesia and postoperative outcome. Lancet 2008;372:2109. author reply 2110-2101.

28. Popping DM, Elia N, Marret E, et al. Protective effects of epidural analgesia on pulmonary complications after abdominal and thoracic surgery: a meta-analysis. Arch Surg 2008; 143:990-999; discussion 1000.

29. Wijeysundera DN, Beattie WS, Austin PC, et al. Epidural anaesthesia and survival after intermediate-to-high risk noncardiac surgery: a population-based cohort study. Lancet 2008;372:562-569.

30. Marret E, Remy C, Bonnet F. Meta-analysis of epidural analgesia versus parenteral opioid analgesia after colorectal surgery. Br J Surg 2007;94:665-673.

31. Werawatganon T, Charuluxanun S. Patient controlled intravenous opioid analgesia versus continuous epidural analgesia for pain after intra-abdominal surgery. Cochrane Database Syst Rev 2005:CD004088.

32. Kong SK, Onsiong SM, Chiu WK, et al. Use of intrathecal morphine for postoperative pain relief after elective laparoscopic colorectal surgery. Anaesthesia 2002;57:1168-1173.

33. Levy BF, Scott MJ, Fawcett W, et al. Randomized clinical trial of epidural, spinal or patient-controlled analgesia for patients undergoing laparoscopic colorectal surgery. Br J Surg 2011; 98:1068-1078.

34. Levy BF, Tilney HS, Dowson HM, et al. A systematic review of postoperative analgesia following laparoscopic colorectal surgery. Colorectal Dis 2010;12:5-15.

35. Virlos I, Clements D, Beynon J, et al. Short-term outcomes with intrathecal versus epidural analgesia in laparoscopic colorectal surgery. Br J Surg 2010;97:1401-1406.

36. Bellomo R, Ronco C, Kellum JA, et al. Acute renal failure-definition, outcome measures, animal models, fluid therapy and information technology needs: the Second International Consensus Conference of the Acute Dialysis Quality Initiative (ADQI). Group Crit Care 2004;8. R204-R212.

37. Holte K, Jensen P, Kehlet H. Physiologic effects of intravenous fluid administration in healthy volunteers. Anesth Analg 2003; 96:1504-1509. 
38. Macafee DA, Allison SP, Lobo DN. Some interactions between gastrointestinal function and fluid and electrolyte homeostasis. Curr Opin Clin Nutr Metab Care 2005;8: 197-203.

39. Srinivasa S, Singh SP, Kahokehr AA, et al. Perioperative fluid therapy in elective colectomy in an enhanced recovery programme. ANZ J Surg 2012;82:535-540.

40. Hubner M, Schafer M, Demartines N, et al. Impact of restrictive intravenous fluid replacement and combined epidural analgesia on perioperative volume balance and renal function within a fast track program. J Surg Res 2010;173:68-74.

41. Srinivasa $S$, Taylor MH, Singh PP, et al. Randomized clinical trial of goal-directed fluid therapy within an enhanced recovery protocol for elective colectomy. Br J Surg 2013;100:66-74.
42. Gustafsson UO, Hausel J, Thorell A, et al. Adherence to the enhanced recovery after surgery protocol and outcomes after colorectal cancer surgery. Arch Surg 2011;146:571-577.

43. Maessen J, Dejong $\mathrm{CH}$, Hausel J, et al. A protocol is not enough to implement an enhanced recovery programme for colorectal resection. Br J Surg 2007;94:224-231.

44. Vlug MS, Wind J, Hollmann MW, et al. Laparoscopy in combination with fast track multimodal management is the best perioperative strategy in patients undergoing colonic surgery: a randomized clinical trial (LAFA-study). Ann Surg 2011;254:868-875.

45. Basse L, Werner M, Kehlet $H$. Is urinary drainage necessary during continuous epidural analgesia after colonic resection? Reg Anesth Pain Med 2000;25:498-501. 